\title{
Compartmentalization in Penicillin G Biosynthesis by Penicillium chrysogenum PQ-96
}

\author{
WIESŁAW KURZĄTKOWSKI ${ }^{1 *}$, MONIKA STANISZEWSKA ${ }^{1}$, MAŁGORZATA BONDARYK $^{1}$ \\ and ANITA GĘBSKA-KUCZEROWSKA²
}

\author{
${ }^{1}$ Independent Laboratory of Streptomyces and Fungi Imperfecti, \\ National Institute of Public Health-National Institute of Hygiene, Warsaw, Poland \\ ${ }^{2}$ Department of Health Promotion and Postgraduate Education, \\ National Institute of Public Health-National Institute of Hygiene, Warsaw, Poland
}

Submitted 13 March 2014, revised 2 October 2014, accepted 13 October 2014

\author{
Abstract
}

The arrangement of organelles in the sub-apical productive non-growing vacuolated hyphal cells of the high- and the low-penicillin-producing strains Penicillium chrysogenum was compared using transmission electron microscopy. In the productive cells of the high-yielding strain the endoplasmic reticulum and the polyribosomes with associated peroxisomes are frequently arranged at the periphery of the cytoplasm and around the vacuoles. At the high activity of penicillin $\mathrm{G}$ biosynthesis the immuno-label of the cytosolic isopenicillin $\mathrm{N}$ synthase is concentrated at the polyribosomes arranged in the peripheral cytoplasm and along the tonoplast as well as around the peroxisomes. On the basis of the obtained results the compartmentalization of the pathway of penicillin G biosymthesis is discussed. The obtained results support the phenylacetic acid detoxification hypothesis of penicillin $\mathrm{G}$ biosynthesis.

Ke y words: Penicillium chrysogenum, biosynthesis, compartmentalization, penicillin G

\section{Introduction}

Penicillin G (Pen G) is a secondary metabolite of Penicillium chrysogenym (Brakhage et al., 2004; Keller etal., 2005; Bartoszewska et al., 2011b; Martin etal., 2012). The biosynthesis of Pen $G$ consists of at least three enzymatic steps. The $\delta$-(L-a-aminoadipyl)-L-cysteinyl$\mathrm{D}$-valine (ACV) synthetase (ACVS) is involved in a nonribosomal condensation of activated precursor amino acids L-a-aminoadipic acid (A), L-cysteine (C) and L-valine (V) to ACV tri-peptide (Weber et al., 2012a). Isopenicillin $\mathrm{N}$ synthase (IPNS) converts the ACV to isopenicillin (IPN). In this oxidative ring closure reaction a bi-cyclic penam nucleus, consisting of a $\beta$-lactam and thiazolidine ring is formed (Weber etal., 2012a, 2012b). The phenylacetyl-CoA ligase (PCL) and the hetero-dimeric acyl-coenzyme A (CoA):6-aminopenicillanic acid (6-APA) acyltransferase (IAT) are involved in the last step of Pen G biosynthesis, in which the a-aminoadipyl group of IPN is substituted by a phenylacetyl side chain (PA) (Weber et al., 2012b).

Localization of the pathway of Pen $\mathrm{G}$ biosynthesis in $P$. chrysogenum cells has been studied using transmis- sion electron microscopy (Kurzątkowski et al., 1991), immuno-gold electron microscopy (Kurzątkowski et al., 1991; Müller et al., 1995; van der Lende et al., 2002a), cell fractionation (Müller et al., 1995; van der Lende et al., 2002a), biochemical and genetic methods (Martin et al., 2012). Nevertheless, at present, the reports concerning the cellular localization of ACVS and IPNS are ambiguous. The enzyme ACVS was previously found to be associated with small membranous organelles (Lendenfeld et al., 1993). Based on its biochemical features, sub-cellular fractionation, immuno-gold electron microscopy localization van der Lende et al. (2002a) suggested that ACVS is a cytosolic enzyme. IPNS appeared to be a soluble cytosolic enzyme (van der Lende etal., 2002a), although its activity in cell free extracts seems to be stimulated by sonification (for review, see van de Kamp et al., 1999) - it suggests a partial compartmentalization of IPNS. This enzyme was also reported to be associated with membranous structures (Kurzątkowski et al., 1991). The enzyme IAT was located in peroxisomes - also called microbodies (Müller et al., 1995; van der Lende et al., 2002a). The cellular organization in Pen G biosynthesis

* Corresponding author: W. Kurzątkowski, Independent Laboratory of Streptomyces and Fungi Imperfecti, National Institute of Public Health-National Institute of Hygiene, Warsaw, Poland; e-mail: wkurzatkowski@pzh.gov.pl 
by high-penicillin-producing strains of $P$. chrysogenum requires further explanation.

This experimental study was designed to provide additional details concerned with the organization of the productive mycelial cells of P. chysogenum at the high activity of Pen G biosynthesis. i.e.: differentiation of hyphal cells, formation and arrangement of peroxisomes, co-location of organelles and enzymes involved in Pen G overproduction.

\section{Experimental}

\section{Materials and Methods}

Strains. In this experimental program the highpenicillin-producing strain P. chrysogenum PQ-96 was examined. Activity of Pen G producing by this strain is presented in Fig. 1. For comparative ultra-structural analyses the low-penicillin-producing strain P. chrysogenum Q-176 was investigated. Pen G production by this strain is presented in Fig. 2.

Cultivation conditions. The tested strains were grown in complex media described previously (Kurząt kowski etal., 1991). Sporulation medium was composed of: saccharose, yeast extract, glycerol, $\mathrm{CaSO}_{4}$ and agar. Inoculated slops of the sporulation medium were cultivated for 7 days at $25^{\circ} \mathrm{C}$. Liquid seed media for growth of biomass of the tested strains contained soy meal and dextrin. The inoculated seed media were incubated in $500-\mathrm{ml}$ flasks for $48 \mathrm{~h}$ at $25^{\circ} \mathrm{C}$ on a rotary shaker (Series 25 Incubator Shaker New Brunswick, Edison, New Jersey, USA) at $250 \mathrm{rpm}$. These cultures were used for inoculation of the production medium which consisted of: corn-steep liquor, glucose and lactose as carbohydrate sources, $\mathrm{CaCO}_{3}$ and salts. The final cultures were incubated in $500-\mathrm{ml}$ flasks at $25^{\circ} \mathrm{C}$ for $72 \mathrm{~h}$ on a rotary shaker at $250 \mathrm{rpm}$. At 8-h intervals an aqueous solution of phenylacetic acid (PA) was added to the cultures at the stationary growth phase. To prevent an overdose during PA administration, the growth of mycelia was observed under a phase-contrast microscope Docuval (Carl-Zeiss, Jena, Germany).

Assay of Pen G. Pen G extraction and analysis were carried out as previously indicated (Garcia-Estrada et al., 2008a).

Transmission electron microscopy. The preparation for a transmission electron microscopy was performed as described previously (Kurzątkowski et al., 1991) with some modifications. The mycelium of a $72-\mathrm{h}$ culture of the tested strains was fixed in $4 \%$ glutaraldehyde (Sigma-Aldrich, Milwaukee, WI, USA) for $20 \mathrm{~h}$ at $3^{\circ} \mathrm{C}$ and then continued with $1 \%$ osmium tetroxide (Sigma-Aldrich, WI, USA) solution for $2 \mathrm{~h}$. The samples were dehydrated in increasing concentrations of ethyl alcohol and embedded in Epon 812 (Serva-Electrophoresis, Heidelberg, Germany). The blocs were polymerized at $37^{\circ} \mathrm{C}$ for $24 \mathrm{~h}$ and at $64^{\circ} \mathrm{C}$ for $48 \mathrm{~h}$, and cut on LKB III Ultra Microtome (Diversified Equipment, Inc., Lorton, VA, USA). The ultrathin sections were stained with uranyl acetate solution and then treated with Reynold's reagent (Serva). The ultrathin sections were examined under a transmission electron microscope JEOL, JEM1220 (Tokyo, Japan).

Antibody production. The IPNS from Cephalosporium acremonium 278 was used in this experimental program (Kurzątkowski et al., 1991). The concentration of the purified enzyme used for immunization was $5 \mathrm{mg}$ in $1 \mathrm{ml}$ of solution. Rabbits were injected four times at 15-days intervals with $500-\mu$ l doses (first injection with a complete Freund's adjuvant and an incomplete adjuvant for the other three injections). The crossreaction of the antibody with the fungal enzyme was confirmed in an enzyme-linked immunosorbent assay and by Western blot analysis.

Processing for immunoelectron microscopy. The methods for immunoelectron microscopy were described previously (Kurzątkowski et al., 1991). The mycelium of a 72-h culture of the high-penicillin-producing strain was washed twice. Agar $(2.5 \%$ w/v Oxoid agar in PBS) coated mycelium was fixed in $0.25 \%$ glutaraldehyde in $\mathrm{PBP}$ at $3^{\circ} \mathrm{C}$ for $15 \mathrm{~h}$. The mycelium was dehydrated with ethanol during the progressive lowering of the temperature conducted in accordance with the following protocol: $30 \%$ ethanol at $0^{\circ} \mathrm{C}$ for $30 \mathrm{~min}$; $50 \%$ ethanol at $-10^{\circ} \mathrm{C}$ for $60 \mathrm{~min} ; 70 \%$ ethanol at $-20^{\circ} \mathrm{C}$ for $60 \mathrm{~min}$; $90 \%$ ethanol at $-35^{\circ} \mathrm{C}$ for $60 \mathrm{~min}$; and $100 \%$ ethanol at $-35^{\circ} \mathrm{C}$ for $90 \mathrm{~min}$ (twice). The infiltration of Lowicryl K4M resin into the mycelia cells was performed at $-35^{\circ} \mathrm{C}$. Low temperature resin polymerization was performed under UV light (nm) for 2 days at $-35^{\circ} \mathrm{C}$ and then continued under UV light for 4 days at room temperature. All steps i.e. dehydration, embedding and polymerization were performed using a freeze substitution apparatus Balzers FSU 010. Ultrathin sections for immuno-labeling were cut with a glass knife on a Reichert OM U3 ultra-microtome. Immunolabeling experiments were carried out at room temperature directly on Lowicryl K4M ultrathin sections resisting on copper grids covered with carbon-coated Pioloform film. The grids were incubated on a drop $(150 \mu \mathrm{l})$ of PBS-glycine solution, $\mathrm{pH}$ 7.4, consisting of $60 \mathrm{mM}$ glycine in PBS for $6 \mathrm{~min}$. Subsequently, the grids were incubated $(3 \times 7 \mathrm{~min})$ on a drop of phosphatebuffered gelatine (PBG) solution, $\mathrm{pH} 7.4$, composed of $0.2 \%(\mathrm{w} / \mathrm{v})$ gelatine and $0.6 \%(\mathrm{w} / \mathrm{v})$ bovine serum albumin in PBS. The rabbit immunoserum to IPNS was diluted 1:500 with PBG and centrifuged at $6000 \mathrm{~g}$ for 2 minutes. The grids with ultrathin sections were incubated on drops of diluted rabbit immunoserum to 

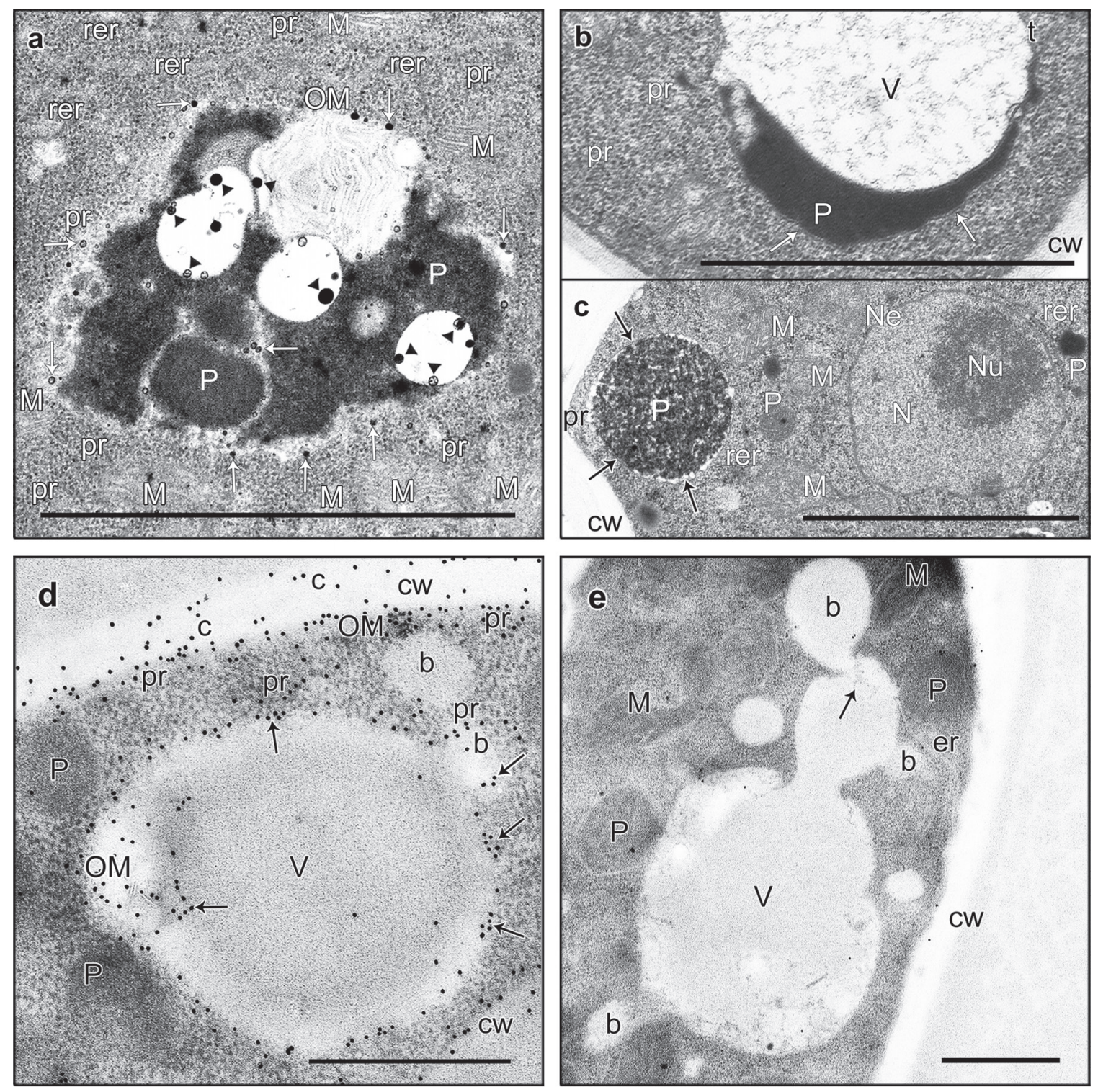

Fig. 1. (a-e) P. chrysogenum PQ-96 (high-yielding strain), $72 \mathrm{~h}$ culture, activity of Pen G biosynthesis (U/ml): total yield at $72 \mathrm{~h}$ of cultivation -8200 , increase of yield between $48 \mathrm{~h}$ and $72 \mathrm{~h}$ of fermentation -4800 ; average increase of yield per one $\mathrm{h}$ between $48 \mathrm{~h}$ and $72 \mathrm{~h}$ of cultivation - 200. Structural organization of the sub-apical metabolically active non-growing vacuolated cell of the hyphae: (a-c) transmission electron microscopy, (d, e) immuno-electron microscopy of IPNS, (d) immuno-gold localization of IPNS, (e) control sample - ultrathin sections treated with rabbit pre-immunoserum, followed by goat anti-rabbit IgG-15 nm gold conjugate. (a) De novo peroxisome (P) formation in association with osmophilic membranes (OM) is seen. Most peroxisomal electron opaque proteins are synthesized on free polyribosomes (pr) and imported directly from the cytosol into the peroxisome (arrous). Note the membrane combined electron opaque proteins concentrated in foci that bud off into the peroxisomal matrix space (arrow heads). The site of peroxisome formation is surrounded by the rough endoplasmic reticulum (rer) and numerous polyribosomes which are accompanied by mitochondria $(\mathrm{M})$. (b, c) Peroxisomes $(\mathrm{P})$ in association with the rough endoplasmic reticulum (rer) and polyribosomes (pr) are visible. (b) The located at the cell wall $(\mathrm{cw})$ large peroxisome $(\mathrm{P})$ consisting of an electron dense protein-reach matrix surrounded by a thin single membrane (arrows) is combined with the tonoplast $(\mathrm{t})$ of the vacuole $(\mathrm{V})$. The peroxisomal volume seems to be an important feature of Pen G production. The increased membrane surface may promote Pen G production as it can maximize the influx of IPN and PA from the cytosol and improve the transport of Pen G from the peroxisome into the cytosol. (c) Peroxisomes (P) from $0.1 \mu \mathrm{m}$ up to $0.6 \mu \mathrm{m}$ in diameter are peripherally located between the cell wall $(\mathrm{cw})$ and the spherical interphase nucleus $(\mathrm{N})$ with a nucleolus $(\mathrm{Nu})$. The peroxisomes are associated with the rough endoplasmic reticulum (rer) and polyribosomes (pr). Note the structural process which might correspond to an initial step of fission inducing membrane curvature that causes peroxisome tubulation followed by organelle scission (arrows). The nuclear envelope (Ne) is associated with the membranes of the rough endoplasmic reticulum and mitochondria (M). (d) Immuno-gold labeling of the cytosolic IPNS (arrows) is mainly located at the periphery of the cytoplasm and around the vacuole $(\mathrm{V})$, and absent in the vacuole as well as in any other organelles. In the cell wall channel-like (c) arranged gold grains can be observed. The peroxisomes (P) are characteristically located at both the cell wall (cw) and the vacuole (V). Vacuolar budding (b) is seen. (e) Immuno-gold electron microscopic localization of not specifically bound antibodies showed exceptionally few gold grains. Note the endoplasmic reticulum (er) which is accompanied by vacuolar budding (b). The mitochondria $(\mathrm{M})$ and the electron transparent massive structure of the cell wall $(\mathrm{cw})$ are visible. Scale bar $=1 \mu \mathrm{m}$. 


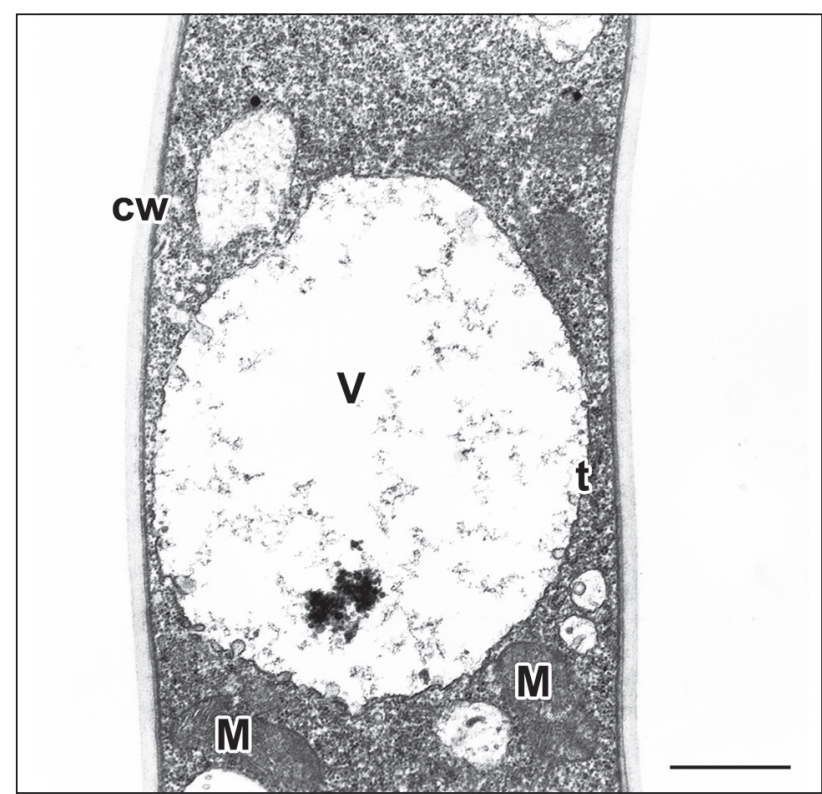

Fig. 2. P. chrysogenum Q-176 (low-yielding strain), $72 \mathrm{~h}$ culture, activity of pen $\mathrm{G}$ production: $80 \mathrm{U} / \mathrm{ml}$. A characteristic feature of the non-growing cells is a lack of large peroxisomes. In the cell a vacuole $(\mathrm{V})$ associated by mitochondria $(\mathrm{M})$ are seen Scale bar $=1 \mu \mathrm{m}$.

IPNS for $35 \mathrm{~min}$ and then washed $(10 \times 30 \mathrm{~s})$ by placing on drops of PBG. Next, the grids were incubated for 30 min on a drop of goat anti-rabbit immunoglobulin $\mathrm{G}$ ( $\operatorname{IgG})-15 \mathrm{~nm}$ gold conjugate (BioCell, Cardiff, UK), diluted 1:25 with PBG. The thin sections were washed $(10 \times 30 \mathrm{~s})$ on PBG drops and $(10 \times 30 \mathrm{~s})$ on PBG-glycine drops. Washing was continued with double-distilled water $(5 \times 30 \mathrm{~min})$. The grids were stained with a $1 \%(\mathrm{w} / \mathrm{v})$ aqueous solution of uranyl acetate for 3 minutes. Finally, the specificity of the immunolabeling procedure was established by performing the following controls: incubation of ultrathin sections with goat anti-rabbit IgG- $15 \mathrm{~nm}$ gold conjugate alone, omitting incubation with the specific antibody; incubation of ultrathin sections with rabbit pre-immunoserum (diluted 1:500 with PBG), followed by goat anti rabbit IgG- $15 \mathrm{~nm}$ goat conjugate. The ultrathin sections were examined under transmission electron microscope JEOL, JEM1220 (Tokyo, Japan).

\section{Results}

At the $72 \mathrm{~h}$ of fermentation the quantity of Pen $\mathrm{G}$ in the broth of the high-yielding strain was about 100 times higher than in the culture of the low-penicillin-producing strain (Fig. 1 and Fig. 2). The antibiotic biosynthesis by the high-yielding strain was accompanied by continuous and intensive PA utilization. In the production medium between $24-72 \mathrm{~h}$ of fermentation a net-like mycelium composed of branching hyphae not damaged by appropriate PA dosage was visible. A remarkable feature is the differentiation of the cells of both strains along the hyphae. Among the 469 cellular sections of the high-yielding strain examined using transmission electron microscopy the apical regions were identified in 29 sections, sub-apical growing hyphal regions in 120 sections, sub-apical non-growing productive vacuolated cells (Fig. 1a-e) in 296 sections, and late-apical degenerating highly vacuolated cells in 24 sections. The ultra-structural differences between these cells can be characterized as follows. The apical $1.0-2.0 \mu \mathrm{m}$ is occupied by a mass of wall vesicles. In median longitudinal sections through the hyphal apex typical tip bodies (Spitzenkörper) composed of small vesicles and groups of ribosomes could be observed (data not shown). The sub-apical growing hyphal regions are densely packed with ribosomes. In the cytoplasm peroxisomes associated with the rough endoplasmic reticulum and numerous mitochondria were visible. A lack of vacuoles and mature cross-walls characterizes these regions (data not shown). In subapical productive non-growing vacuolated cells of the high-yielding strain the polyribosomes are frequently placed at the periphery of the cytoplasm and around the vacuoles. The membranes of the endoplasmic reticulum abundantly transform into numerous peroxisomes from $0.1 \mu \mathrm{m}$ up to $1.0 \mu \mathrm{m}$ in diameter which are accompanied by the rough endoplasmic reticulum and polyribosomes (Figs. 1a-c). De novo synthesis of peroxisomes is shown in Fig. 1a. The collocation of large peroxisome with vacuole (Fig. 1b) and peroxisome multiplication by fission (Fig. 1c) are significant features of the high productive non-growing cells. Massive autophagy was detected predominantly in the late-apical highly vacuolated cells. In these cells autophagosomes could be observed. Autophagy was accompanied by peroxisome and cytoplasm degradation (data not shown). At the high activity of Pen G biosynthesis the immuno-gold location of the cytosolic IPNS was concentrated at the periphery of the cytoplasm and around the vacuoles as well as in channel-like structures situated in the cell wall (Fig. 1d). Peroxisomes accompanied vacuolar budding could be observed (Fig. 1d, e). The immunogold labeling of IPNS was analyzed on 240 sections of the sub-apical productive non-growing vacuolated cells of the high-yielding strain. Control experiments have been performed in order to check the specificity of immuno-gold labeling. The sample with IgG-gold conjugate alone was essentially devoid of labeling. The control incubation with pre-immunoserum, followed by goat anti-rabbit IgG- $15 \mathrm{~nm}$ gold conjugate exhibited only very light labeling of the cell (Fig. 1e). In the mature non-growing hyphal cells of the low-penicillinproducing strain P. chrysogenum Q-176 a lack of peroxisomes above $0.1 \mu \mathrm{m}$ in diameter could be observed 

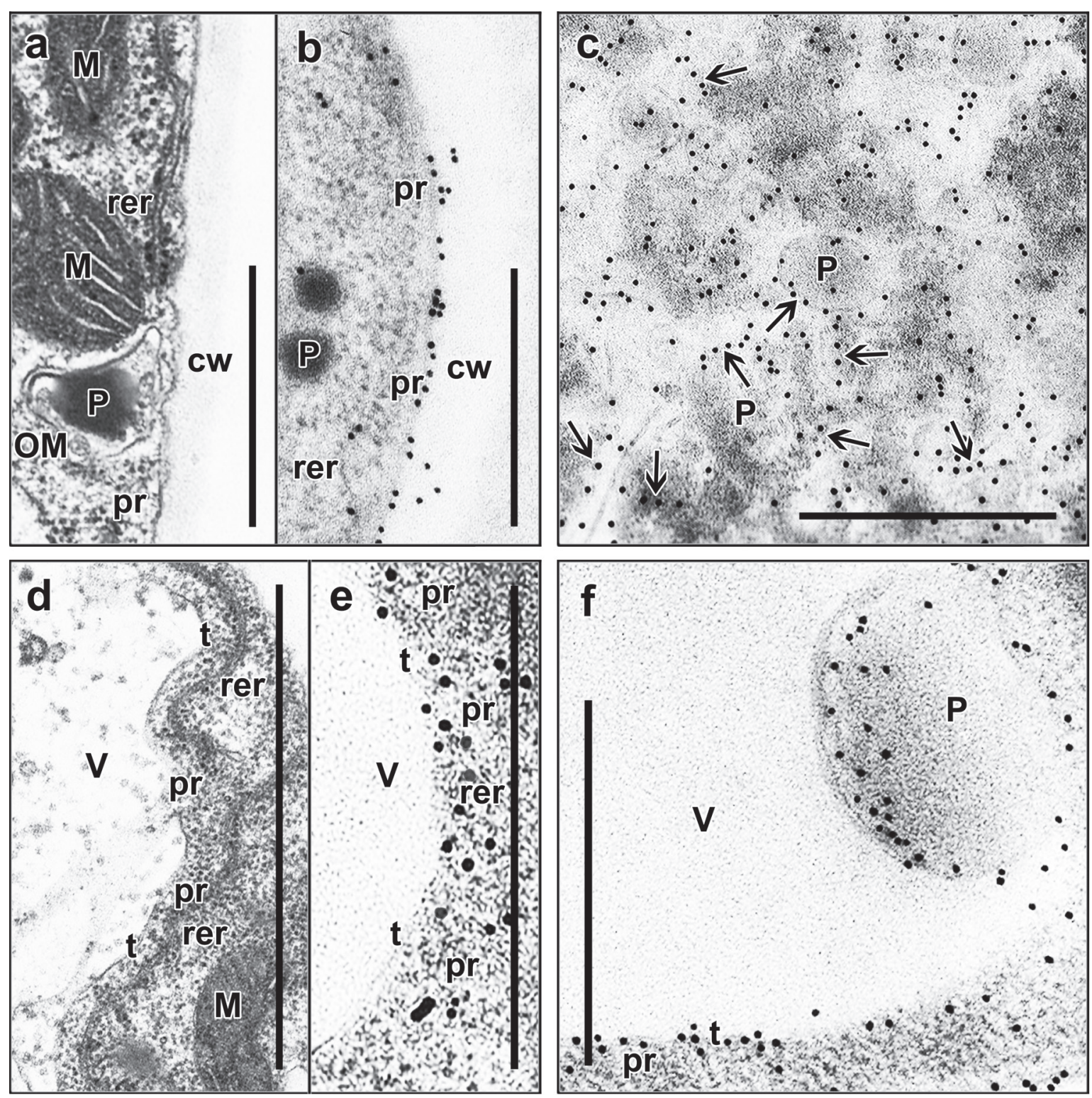

Fig. 3. (a, f) P. chrysogenum PQ-96 (high-yielding strain), 72-h of cultivation, structural organization of the sub-apical metabolically active non-growing vacuolated cell of the hyphae: (a, d) transmission electron microscopy, (b, c, e, f) immuno-gold electron microscopy of IPNS. (a) At the periphery of the cytoplasm close to the cell wall (cw) an osmophilic membrane (OM) embedded peroxisome is visible $(\mathrm{P})$. The peroxisome is associated with the rough endoplasmic reticulum (rer) and polyribosomes (pr) which are accompanied by mitochondria (M). (b) The immuno-gold labeling of the cytosolic IPNS is concentrated at the polyribosomes (pr) situated in the peripheral cytoplasm close to the rough endoplasmic reticulum (rer) and peroxisomes (P). (c) Surface section of the hypha showing the linear arranged imuno-gold marker of IPNS (arrows) adhering to the membranes surrounding the cross sectioned peroxisomes (P). (d) Note the osmophilic membranes of the rough endoplasmic reticulum (rer) and polyribosomes (pr) located along the tonoplast (t) of the vacuole $(\mathrm{V})$. Such a cellular organization is a characteristic feature of the sub-apical non-growing vacuolated cells at the high activity of Pen G biosynthesis. (e) The immuno-gold marker of IPNS is abundantly adhering to the polyribosomes (pr) located in the vicinity of the vacuolar $(\mathrm{V})$ tonoplast $(\mathrm{t})$. (f) Note the immuno-gold marker of IPNS linearly arranged around the peroxisome (P) and along the tonoplast $(\mathrm{t})$ that is accompanied by polyribosomes (pr). During high activity of Pen G biosynthesis by the productive non-growing cells the peroxisomes engulfed by the vacuolar membrane showed frequently a structure not degraded by the vacuolar hydrolases. Scale bar $=1 \mu \mathrm{m}$.

(Fig. 2). This strain tolerated only $10 \%$ of PA that was added to the fermentation broth of the high-yielding strain. An overdose of PA resulted in extensive damages of the mycelial cells. Small peroxisomes were also rarely visible in the cytoplasm of the low-penicillin-producing strain Aspergillus niger (data not shown).
Further comparative studies of the sub-apical nongrowing productive vacuolated cells of the highyielding strain using transmission electron microscopy and immunoelectron microscopy are presented in Fig. 3 (a-f). These studies exhibit the accumulation of the cytosolic IPNS at both the rough endoplasmic 


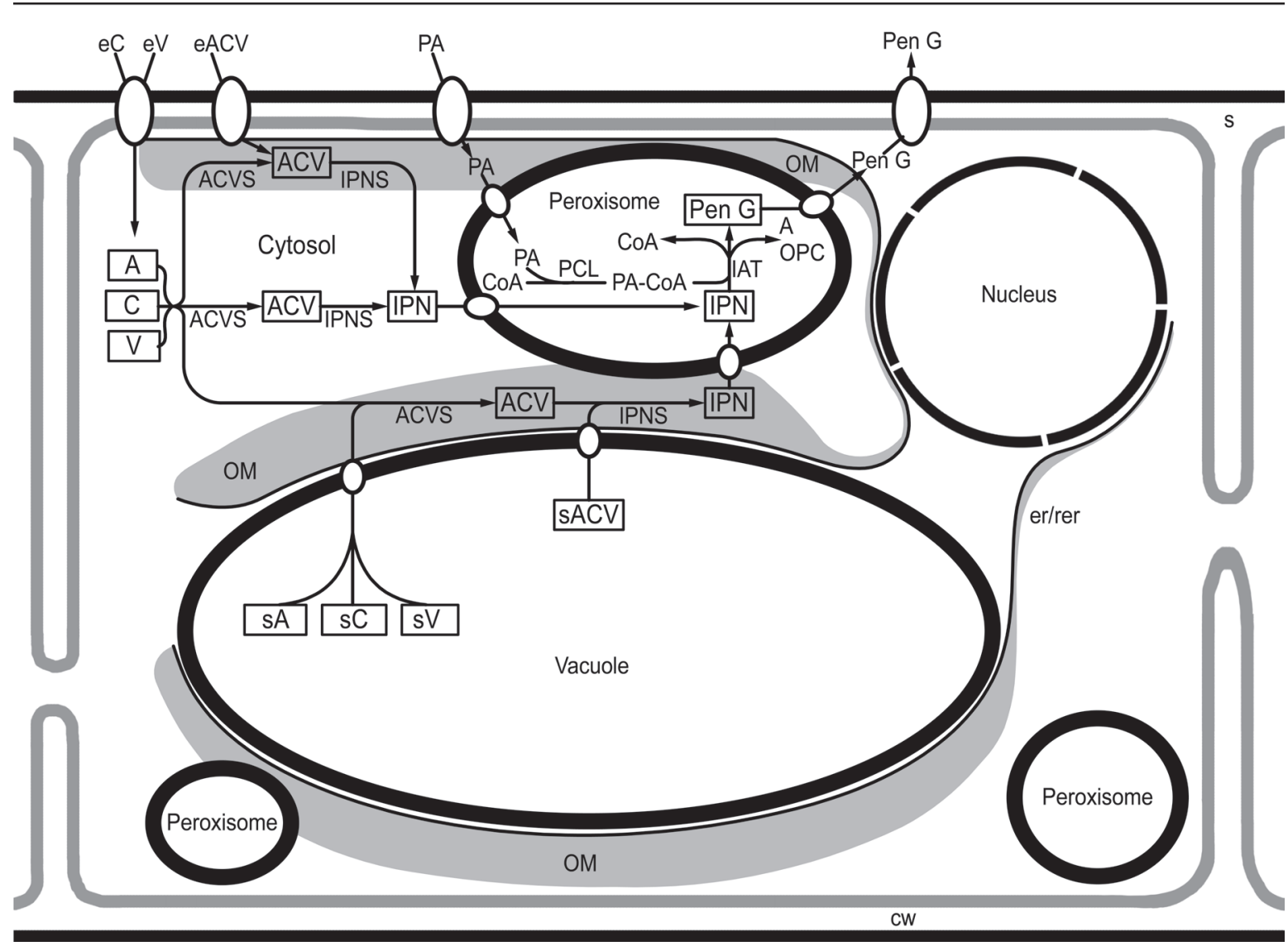

Fig. 4. A schematic overview of the pathway of Pen G biosynthesis compartmentalized in sub-apical productive non-growing vacuolated hyphal cells of P. chrysogenum PQ-96 (high pen G yielding strain) at high activity of Pen G production. Note the aggregated organelles building the assembly line of Pen G biosynthesis. The arrangement of the osmophilic membranes (OM) of the endoplasmic reticulum (er/rer) at the periphery of the cytoplasm and around the vacuoles is a significant ultrastructural feature in Pen G biosynthesis. The osmophilic membranes transform abundantly into peroxisomes. The ACVS and IPNS distributed over the cytosol and concentrated at both the rough endoplasmic reticulum (rer) and polyribosomes surrounding the peroxisomes can be sufficiently supplied with precursor amino acids and the tri-peptide intermediate from the cytosol ( $\mathrm{A}, \mathrm{C}, \mathrm{V}, \mathrm{ACV}$ ) as well as from the medium (eC, eV, eACV). Moreover, the sequestered into a vacuolar pool precursor amino acids $\mathrm{sA}, \mathrm{sC}, \mathrm{sV}$ and the intermediate sACV may play an ancillary supplying role in Pen G biosynthesis. The intermediate IPN is transported from the cytosol into the peroxisome where the two enzymes PCL and IAT involved in the last steps of Pen G biosynthesis are located. Some putative enzymatic and transport steps building the assembly lines of Pen G biosynthesis are depictured by arrows. Abbreviations: (cw) cell wall, (s) septum.

reticulum and polyribosomes abundantly arranged along the cytoplasm membrane and at the tonoplast as well as around the associated peroxisomes. Models depicting the cell biology in Pen G biosynthesis by P. chrysogenum PQ-96 including some putative transport steps are depicted in Fig. 4.

\section{Discussion}

In our study the organization of mycelial cells of the high- and the low-yielding strains at the high activity of Pen $\mathrm{G}$ biosynthesis was compared. These findings, based on the analyses of a large number of ultrathin sections examined using transmission electron microscopy and immuno-gold localization of IPNS, provide new details regarding the compartmentalization in Pen $\mathrm{G}$ biosynthesis. The overproduction of Pen $\mathrm{G}$ was associated with a strictly adjusted cellular organization. The productive mycelia cells of the high-yielding strain exhibited numerous large peroxisomes frequently arranged at the periphery of the cytoplasm and around the vacuoles. Using the method of immuno-gold labeling of IAT such a characteristic arrangement of peroxisomes was also visible in the cells of the high-yielding strain of P. chrysogenum (van der Lende et al., 2002a; Garcia-Estrada et al., 2008a). Our findings suggest that at the high activity of Pen G biosynthesis IPNS is accumulated at the place of its biosynthesis, i.e. at polyribosomes frequently situated in the peripheral cytoplasm and around the vacuoles, including the polyribosomes surrounding the peroxisome encompassed IAT and PCL. Such an arrangement of peroxisomes may increase the IAT and PCL supplying efficacy in Pen G biosynthesis from the medium and from the cytosol as well as from the vacuolar pool. Moreover, the increased cellular 
vacuolization caused by peroxisome associated vacuolar budding seems to be involved in Pen G biosynthesis. Our findings suggest that in Pen G biosynthesis the structurally grouped organelles build a well organized "assembly line" composed of cytosol concentrated and membrane encompassed enzymes, substrates, intermediates, precursors, side- and end-products. Such a cellular organization was not visible in the mature cells of the low-penicillin-producing strain Q-176. This finding seems hardly to confirm our remarks concerning the cellular organization in Pen G biosynthesis. Our discoveries are consistent with the reported positive correlation between the number of large peroxisomes and Pen G biosynthesis (Kiel et al., 2005; 2009; Meijer et al., 2010; Nagotu et al., 2010), as well as between the extend of vacuolization and antibiotic production (Paul and Thomas, 1996). It was also reported that the vacuole may play an auxiliary role in the supply of precursor amino acids, and in the storage of intermediates in Pen $\mathrm{G}$ biosynthesis (Lendenfeld et al., 1993; van de Kamp et al., 1999). Our observations of the high-yielding mature cells of the mycelium exhibited both the de novo formation of large peroxisomes from the endoplasmic reticulum and peroxisome fission. In accordance with our results, two models of peroxisome development have been documented, namely de novo synthesis from the endoplasmic reticulum and multiplication by fission of pre-existing organelles (Hoepfner et al., 2005; Tam et al., 2005; Motley and Hettema, 2007; Jourdain et al., 2008; Motley et al., 2008; Perry et al., 2009; Meijer et al., 2010; Bartoszewska et al., 2011a; 2011b; Nuttall et al., 2011; Opaliński et al., 2012).

Based on the biochemical features van de Kamp et al. (1999) concluded that ACVS is predominantly located in the cytosol. A more recent cell fractionation under conditions that prevent proteolysis and immunogold electron microscopy localization indicated that ACVS is a cytosolic enzyme (van der Lende et al., 2002a). This finding is in accordance with the active status of ACVS in Pen G biosynthesis. Purified ACVS has a sharp pH optimum above 7.5 (Theilgaard et al., 1997) corresponding with the cytosolic $\mathrm{pH}$ of approx. 7.0-7.6. We suggest, that the cytosolic enzyme ACVS of the high-yielding strain is collocated with IPNS and accumulated at the polyribosomes. It is in accordance with the reports concerning a membrane-associated location of ACVS from P. chrysogenum. For example, with the help of antibodies against ACVS, Müller et al. (1995) detected ACVS in a 100,000-g pellet that contains small membranous structures. They indicated that tri-peptide formation is a membrane-attached process. On the bases of marker enzyme activities, Lendenfeld et al. (1993) assigned the 100,000- $g$ pellet fraction to vacuolar vesicles. This study pointed to a loose attachment of ACVS to membranes.
The intracellular precursor amino acids concentration is important in view of the affinity of ACVS for its substrates. ACVS from P. chrysogenum has affinities in sub-mM range, i.e.: $46 \mu \mathrm{M}$ for $\mathrm{A}, 80 \mu \mathrm{M}$ for $\mathrm{C}$ and $83 \mu \mathrm{M}$ for $\mathrm{V}$. The above suggested accumulation of ACVS in the productive cells of the high-yielding strain enables its continuous and efficient supplying with precursor amino acids directly from the cytosol and vacuole as well as from the fermentation broth. In accordance with this argumentation, it was described that in $P$. chrysogenum these precursor amino acids are sequestered from the cytosol into a vacuolar pool. It was also indicated that the precursor amino acids are withdrawn specifically from the vacuolar pool (van der Lende, 2002a). An exchange between cytosolic and vacuolar pools may play an important role in Pen G biosynthesis by $P$. chrysogenum (Lendenfeld et al., 1993; van der Lende, 2002a). Total intracellular concentrations of $\mathrm{A}, \mathrm{C}$ and $\mathrm{V}$ are approx. $<0.1-1.4,0.1-0.4$ and 1.1-2.4 mM. A positive correlation exists between the extracellular precursor amino acids concentration and Pen $G$ production. The transport of precursor amino acids from the fermentation medium through the plasma membranes into the cytoplasm was reviewed by van de Kamp et al. (1999). Uptake of amino acids from the medium may contribute to Pen G biosynthesis (van de Kamp et al., 1999). Special additions to the medium contain amino acids. An example is corn-steep liquor that is used for Pen G biosynthesis by P. chrysogenum which contains $\mathrm{C}$ and $\mathrm{V}$.

Fractionation experiments clearly demonstrate that IPNS is a soluble cytosolic protein (Müller et al., 1995). Using immuno-gold electron microscopy, IPNS was localized in the cytosol (van der Lende at al., 2002a). It was also reported that IPNS activity in cell free extracts seemed to be stimulated by the addition of Triton X-100 or by sonification (for review, see van de Kamp et al., 1999). It suggests the partial compartmentalization of IPNS in the cell. The surveys of a large number of hyphal sections leads us to the conclusions that IPNS may abundantly appear between polyribosomes and peroxisomes abundantly arranged at the periphery of the cell and along the tonoplast. For immuno-gold localization of IPNS, the preservation of the protein antigenicity and ultra-structure of the tested strain are prerequisites for a sensitive and accurate immunogoldlabeling. Fixation with $0.25 \%$ glutaraldehyde, dehydration by the technique involving progressive lowering of temperature, low-temperature embedding into Lowicryl K4M proved to be suitable procedures employed in our experimental program. After treatment of ultrathin sections with antibodies to IPNS and IgG gold conjugates a labeling pattern was obtained. The conducted control experiments ascertain the specificity of immunogold-labeling. 
The suggested cellular accumulation of IPNS may enhance its selective, continuous and sufficient substrate supply in Pen $\mathrm{G}$ biosynthesis. In line with this argumentation, it was recently found that ACV is present in the cytosol and vacuoles and accumulates in the medium to concentrations of up to $2 \mathrm{~mm}$ (Lendenfeld et al., 1993). The intracellular ACV content during high-level Pen $\mathrm{G}$ production by P. chrysogenum increases from 0.25 to $4.0-6.5 \mathrm{~mm}$. The affinity of IPNS for ACV is in the sub-mM range (for review, see van de Kamp et al., 1999). The high concentration of ACV in the fermentation broth might explain our unexpected results concerning the localization of IPNS at the periphery of the cytoplasm and in the channel-like lamellar structures of the cell wall. Such a location might be a precisely adopted structural arrangement enabling the withdrawal of ACV immediately from the fermentation broth and from the cytoplasm for the peripherally located IPNS to increase the efficacy and yield in Pen G biosynthesis.

The localization of IAT in peroxisomes was shown by immuno-gold labeling in combination with transmission electron microscopy performed on whole cells and on sub-cellular fractions enriched in peroxisomes (Garcia-Estrada et al., 2008a, 2008b; Müller et al., 1995; van der Lende et al., 2002a). This solid evidence was also confirmed by biochemical data (Martin et al., 2012). PCL and IAT both harbor a peroxisomal type 1 targeting signal (PTS1) at the C-terminus (for review, see van de Kamp et al., 1999). The $\mathrm{pH}$ of the lumen of the peroxisomes of $P$. chrysogenum is in the range of $\mathrm{pH}$ $7.5-8.0$ and is comparable with the $\mathrm{pH}$ optima of IAT and PCL of 8.0 and 8.5, respectively (van der Lende, 2002b; Koetsier et al., 2009). The advantages for peroxisomal compartmentalization of IAT and PCL could be a higher concentration of both enzymes and substrates, as well as possibility of preventing the loss of metabolic intermediates (Evers et al., 2004).

During high-level Pen G production by P.chrysogenum the intracellular IPN concentration is in the range of $0.5-1.0 \mathrm{~mm}$. IPN does not accumulate in the medium, its extracellular concentration remains approx. constant at $0.2-0.4 \mathrm{~mm}$. It was suggested that the IPN substrate of IAT might be regained from the fermentation broth via transport across the plasma membrane. The affinity of the peroxisome-located IAT from P. chrysogenum for IPN lies in the $\mu \mathrm{M}$-range and for PA-CoA is $6.0 \mu \mathrm{M}$ (for review, see van de Kamp et al., 1999). Our findings indicate that IPN transport for IAT results abundantly from the IPNS entrapped between the polyribosomes and the peroxisomal membranes. PA is supplied for Pen $\mathrm{G}$ biosynthesis from the fermentation broth across the plasma membrane. It was concluded, that during Pen G biosynthesis the main PA flow across the plasma membrane and the peroxisomal membrane occurs through passive diffusion (Hillenga et al., 1995; Weber etal., 2012a). Depending on the external concentration, its intracellular accumulation ranges up to hundreds of $\mathrm{mM}$ (Hillenga et al., 1995).

Classical strain improvement has yielded industrial P. chrysogenum strains that produce high titers of Pen G. These strains contain up to eight copies of the Pen G biosynthetic gene cluster consisting of three genes $p c b A B$, $p c b C$ and penDE encoding the key enzymes ACVS, IPNS and LAT, respectively. The PCL enzyme encoded by $p h l$ gene is not a part of the Pen G biosynthetic gene cluster. Numerous genomic and transcriptional analysis of strain lineages have led to the identification of several essential alterations in high-yielding strains, including the amplification of the Pen G biosynthetic gene cluster, elevated transcription of genes involved in biosynthesis of this antibiotic and its amino acid precursors, and genes encoding peroxisome proliferations (for a review, see Weber et al., 2012a; 2012b). In P. chrysogenum, the biosynthetic pathway of Pen G is compartmentalized (Evers et al., 2004; Martin et al., 2010) and is suggested to take place in sub-apical, productive non-growing vacuolated hyphal cells (Paul and Thomas, 1996). Our findings allow suggest that in the productive cells of the high Pen $G$ yielding strain the cytosolic enzymes ACVS and IPNS are concentrated at the peroxisome encompassed IAT and PCL. It implies that ACV can be immediately oxidized to IPN (van der Lende et al., 2002a), which is important since ACVS appears to be a relatively inefficient enzyme with a low turnover value and IPNS is rather instable in the presence of ACV (Perry et al., 2009). The aggregation of peroxisomes at the periphery of the cytoplasm and around the vacuoles where ACVS and IPNS are suggested to be collocated may facilitate the continuous and efficient biosynthetic flow and the immediate conversions in Pen G biosynthesis. The formation of the $\beta$-lactam nucleus takes place in the cytosol. IPN is subsequently transported into the adjacent peroxisomes where the hydrophilic L- $\alpha$-aminoadipyl side chain of IPN is exchanged for a hydrophobic phenylacetyl group of the side chain precursor by IAT (van der Lende et al., 2002a; Weber et al., $2012 \mathrm{a} ; 2012 \mathrm{~b}$ ). The side chain precursor has to be activated before the transacylation occurs. This reaction is catalyzed by the peroxisomal PCL (Meijer et al., 2010). It was suggested, that the peroxisomal collocation of IAT and PCL provides a clear benefit for the coordination of the last step in Pen G biosynthesis (Martin et al., 2012). Peroxisomes play a crucial role in the biosynthesis of Pen G by P. chrysogenum (Müller et al., 1995). High Pen $G$ producing strains show increasing numbers of peroxisomes (van den Berg et al., 2008; Meijer et al., 2010; Weber et al., 2012a). Mutants blocked in the biosynthesis of peroxisomes have a significantly reduced Pen V production (Weber et al., 2012a). Overexpression of the peroxisome proliferation gene pex 11 
in $P$. chrysogrnum results in an increase of both peroxisome numbers and the activity of PenV biosynthesis (Kiel et al., 2005). When the PTS1 targeting signal is removed from IAT, the enzyme no longer localizes the peroxispmes, but is diverted to the cytosol and the vacuole. In these cells, Pen G production by P. chrysogenum is completely abrogated (Weber et al., 2012a). The presence of functional peroxisomes is important for the efficiency of $\beta$-lactam antibiotics production by $P$. chrysogenum (Kiel etal., 2005; van den Berg etal., 2008; Meijer et al., 2010; Opaliński et al., 2010; Bartoszewska et al., 2011a; 2011b; Opaliński et al., 2012). A constitutive autophagy-related degradation of peroxisomes and cytosolic compartments was observed in late sub-apical cells of $P$. chrysogenum under penicillin production conditions. The inactivation of this processes by disruption of ATG gene encoding a serine-threonine kinase results in enhanced IAT and IPNS levels and in significantly increased production of Pen V (Meijer et al., 2010; Bartoszewska et al., 2011a). Metabolic engineering has proven to be a rational alternative to classical strain improvement (Thykaer and Nielsen, 2003). So far, it is unknown whether IPN and Pen G transport over the peroxisomal membrane requires active transport or utilizes peroxisomal pore proteins (Antonenkov and Hiltunen, 2012). Secretion of Pen G across the plasma membrane of $P$. chrysogenum is only poorly understood (Weber et al., 2012a). The extracellular level of Pen G is tenfold higher than the intracellular concentration (van den Berg et al., 2008), which suggest an active secretion mechanism (for revive, see Weber et al., 2012a).

In high concentration PA is toxic to the mycelial cells (Martin et al., 2012). During industrial production of Pen G, PA is fed in small amounts to the medium to avoid toxic side effects (Hillenga et al., 1995). The fungal cytoplasmic acidification results in the inhibition of enzymatic reactions and amino acid transport, leading to cell death. PA is taken up from the medium and in the peroxisomes coupled with 6-APA. We suggest that the numerous large peroxisomes arranged at the periphery of the cytoplasm and at the vacuoles seem to build a PAbinding target protecting the cell from the toxicity of the precursor of Pen $G$ biosynthesis. It suggests that Pen $G$ biosynthesis in the industrial scale is involved in a cellular detoxification process. In accordance with this suggestion, it was described that the formation of secondary metabolites has implications for various cellular processes, including cellular defense (Keller et al., 2005). In this process peroxisomes mainly perform detoxification reactions of toxic to the cytoplasm organic compounds and metabolites leading to the biosynthesis of secondary metabolites with interesting biological or pharmacological activities (Martin et al., 2012). Peroxisomes are dynamic organelles that often proliferate in response to compounds that they metabolize (Tam et al., 2005).
The novelty of this experimental program is the discovery of essential cellular features in Pen G biosynthesis by P. chrysogenum PQ-96. These findings allow suggest a phenylacetic acid detoxification hypothesis of Pen G production. The sub-apical non-growing peroxisomal cells of the mycelium of the high-yielding strain are privileged in the overproduction of Pen G. The antibiotic biosynthesis by the high-yielding strain is associated with numerous large de novo synthesized peroxisomes which are abundantly arranged at the plasma membrane and in the neighborhood of the tonoplast. The cytosolic enzyme IPNS is accumulated between the polyribosomal membranes and the peroxisomes. Such an arrangement increases the peroxisomal IAT and PCL supplying efficacy in Pen G biosynthesis from the medium and from the cytosol as well as from the vacuolar pool.

\section{Acknowledgements}

This work was supported by the grants from the Polfa Tarchomin Pharmaceutical Works, Warsaw; Institute of Biochemistry and Molecular Biology, Berlin; Robert Koch-Institute, Berlin. We thank H. Palissa, H. Van Liempt, H. Von Döhren, H. Kleinkauf and W.P. Wolf for the initiation and assistance of the immunogold localization of IPNS.

\section{Literature}

Antonenkov V.D. and J.K. Hiltunen. 2012. Transfer of metabolites across the peroxisomal membrane. Biochim. Biophys. Acta 1822: 1374-1386.

Bartoszewska M., J.A. Kiel, R.A. Bovenberg, M. Veenhuis and I.J. van der Klei. 2011a. Autophagy deficiency promotes $\beta$-lactam production in Penicillium chrysogenum. Appl. Environ. Microbiol. 77: 1413-1422.

Bartoszewska M., Ł. Opaliński, M. Veenhuis and I.J. van der Klei. 2011b. The significance of peroxisomes in secondary metabolite biosynthesis in filamentous fungi. Biotechnol. Lett. 33: 1921-1931. Brakhage A.A., P. Spröte, Q. Al-Abdallah, A. Gehrke, K. Plaftner and A. Tüncher. 2004. Regulation of penicillin biosynthesis in filamentous fungi. Adv. Biochem. Eng. Biotechnol. 88: 45-90.

Evers M.E., H. Trip, M. A. van den Berg, R.A. Bovenberg and A.J. Driessen. 2004. Compartmentalization and transport in $\beta$-lactam antibiotics biosynthesis. Adv. Biochem. Eng. Biotechnol. 88: 111-135.

Garcia-Estrada C., I. Vaca, F. Fierro, K. Sjollema, M. Veenhuis and J.F. Martin. 2008a. The unprocessed preprotein from IATC ${ }^{1035}$ of the isopenicillin $\mathrm{N}$ acyltransferase is transported inside peroxisomes and regulates its self-processing. Fung. Genet. Biol. 45: 1043-1052.

Garcia-Estrada C., I. Vaca and M. Lamas-Maceiras. 2008b. In vivo transport of the intermediates of the penicillin biosynthetic pathway in tailored strains of Penicillium chrysogenum. Appl. Microbiol. 76 169-182.

Hillenga D.J., H.J. Versantvoort, S. van der Molen, A.J. Driessen and W.N. Konings. 1995. Penicillium chrysogenum takes up the penicillin $\mathrm{G}$ precursor phenylacetic acid by passive diffusion. Appl. Environ. Microbiol. 61: 2589-2595.

Hoepfner D., D. Schildknegt, I. Braakman, P. Philippsen and H.F. Tabak. 2005. Contribution of the endoplasmic reticulum to peroxisome formation. Cell. 122: 85-95. 
Jourdain I., D. Sontam, C. Johnson, C. Dillies and J.S. Hyams. 2008. Dynamin-dependent biogenesis cell cycle regulation and mitochondrial association of peroxisomes in fission yeast. Traffic 9: 353-365.

Keller N.P., G. Turner and J.W. Bennett. 2005. Fungal secondary metabolism-from biochemistry to genomics. Nat. Rev. Microbiol. 3: 937-947.

Kiel J.A., M.A. van den Berg, F. Fusetti, B. Poolman, R.A. Bovenberg, M. Veenhuis and I.J. van der Klei. 2009. Matching the proteome to the genome: the microbody of penicillin-producing Penicillium chrysogenum cells. Funct. Integr. Genomics 9: 167-184.

Kiel J.A., I.J. van der Klei, M.A. van den Berg, R.A. Bovenberg and M. Veenhuis. 2005. Overproduction of a single protein, PcPex11p, results in 2-fold enhanced penicillin production by Penicillium chrysogenum. Fungal. Genet. Biol. 42: 154-164.

Koetsier M.J., P.A. Jekel, M.A. van den Berg, R.A. Bovenberg and D.B. Jenssen. 2009. Characterization of a phenylacetate - CoA ligase from Penicillium chrysogenum. Biochem. J. 417: 467-476.

Kurzątkowski W., H. Palissa, H. Van Liempt, H. von Döhren, H. Kleinkauf, W.P. Wolf and W. Kuryłowicz. 1991. Localization of isopenicillin N synthase in Penicillium chrysogenum PQ-96. Appl. Microbiol. Biotechnol. 35: 517-520.

Lendenfeld T., D. Ghali, M. Wolschek, E.M. Kubicek-Pranz and C.P. Kubicek. 1993. Subcellular compartmentation of penicillin biosynthesis in Penicillium chrysogenum - the amino acids are precursors derived from the vacuole. J. Biol. Chem. 268: 665-671.

Martin J-F., R.V. Ullán and C. Garcia-Estrada. 2010. Regulation and compartmentalization of $\beta$-lactam biosynthesis. Microb. Biotechnol. 3: 285-299.

Martin J-F., Ullán R.V. and C. Garcia-Estrada. 2012. Role of peroxisomes in the biosynthesis and secretion of $\beta$-lactams and other secondary metabolites. J. Ind. Microbiol. Biotechnol. 39: 367-382.

Meijer W.H., L. Gidijala, S. Fekken, J.A. Kiel, M.A. van den Berg, R. Lascaris, A.L. Roal, R.A. Bovenberg and I.J. van der Klei. 2010. Peroxisomes are required for efficient penicillin biosynthesis in Penicillium chrysogenum. Appl. Environ. Microbiol. 76: 5702-5709. Motley A.M. and E.H. Hettema. 2007. Yeast peroxisomes multiply by growth and division. J. Cell. Biol. 178: 399-410.

Motley AM, G.P. Ward and E.H. Hettema. 2008. Dnm1p-dependent peroxisome fission requires Caf4p, Mdv1p and Fis1p. J. Cell. Sci. 121: 1633-1640.

Müller W.H., J. Essers, B.M. Humbel and A.J. Verkleij. 1995. Enrichment of Penicillium chrysogenum microbodies by isopycnic centrifugation in nycodenz as visualized with immuno-electron microscopy. Biochem. Biophys. Acta 1245: 215-220.

Nagotu S., M. Veenhuis and I.J. van der Klei. 2010. Divide et impera: The dictum of peroxisomes. Traffic 11: 175-184.

Nuttall J.M., A. Motley and E.H. Hettema. 2011. Peroxisome biogenesis recent advances. Curr. Opin. Cell. Biol. 23: 421-426.
Opaliński Ł, J.A. Kiel, T.G. Homan, M. Veenhuis and I.J. van der Klei. 2010. Penicillium chrysogenum Pex14/17p - a novel component of the peroxisomal membrane that is important for penicillin production. FEBS J. 277: 3203-3218.

Opaliński Ł., M. Bartoszewska, S. Fekken, H. Liu, R. de Boer, I. van der Klei, M. Veenhuis and J.A. Kiel. 2012. De Novo peroxisome biogenesis in Penicillium chrysogenum is not dependent on the Pex11 family members or Pex16. PLoS ONE 7: e35490, DOI: 10.1371/journal.pone.0035490.

Paul G.C. and C.R. Thomas. 1996. A structured model for hyphal differentiation and penicillin production using Penicillium chrysogenum. Biotechnol. Bioeng. 51: 558-572.

Perry R.J., F.D. Mast and R.A. Rachubiński. 2009. Endoplasmic reticulum-associated secretory proteins Sec20p, Sec39p and Dsl1p are involved in peroxisome biogenesis. Eukaryot. Cell. 8: 830-843. Tam Y.Y., A. Fagarasanu, M. Fagarasanu and R.A. Rachubiński. 2005. Pex3p initiates the formation of a preperoxisomal compartment from a subdomain of the endoplasmic reticulum in Saccharomyces cerevisiae. J. Biol. Chem. 280: 34933-34939.

Theilgaard H.B., K.N. Kristiansen, C.M. Henriksen and J. Nielsen. 1997. Purification and characterization of $\delta$-(L- $\alpha$-aminoadipyl)-Lcysteinyl-D-valine synthetase from Penicillium chrysogenum. Biochem. J. 327: 185-191.

Thykaer J. and J. Nielsen. 2003. Metabolic engineering of $\beta$-lactam production. Metab. Eng. 5: 56-69.

van de Kamp M., A.J. Driessen and W.N Konings. 1999. Compartmentalization and transport in $\beta$-lactam antibiotic biosynthesis by filamentous fungi. Antonie van Leeuwenhoek 75: 41-78.

van den Berg M.A., R. Albang, K. Albermann, J.H. Badger, J-M. Daran, A.J. Driessen, C. Garcia-Estrada, N.D. Fedorova, D.M. Harris, W.H. Heijne and others. 2008. Genome sequencing and analysis of the filamentous fungus Penicillium chrysogenum. Nat. Biotechnol. 26: 1161-1168.

van der Lende T.R., M. van de Kamp, M. van den Berg, K. Sjollema, R.A. Bovenberg, M. Veenhuis, W.N. Konings and A.J. Driessen. 2002a. $\delta$-(L-a-Aminoadipyl)-L-cysteinyl-D-valine synthetase, that mediates the first committed step in penicillin biosynthesis, is a cytosolic enzyme. Fungal. Genet. Biol. 37: 49-55.

van der Lende T.R., P. Breeuwer, T. Abee, W.N. Konings and A.J. Driessen. 2002b. Assessment of the microbody luminal pH in the filamentous fungus Penicillium chrysogenum. Biochim. Biophys. Acta 1589: 104-111.

Weber S.S., R.A. Bovenberg and A.J. Driessen. 2012a. Biosynthetic concepts for the production of $\beta$-Lactam antibiotics in Penicillium chrysogenum. Biotechnol. J. 7: 225-236.

Weber S.S., F. Polli, R. Boer, R.A. Bovenberg and A.J. Driessen. 2012b. Increased penicillin production in Penicillium chrysogenum strains via balanced overexpression of isopenicillin $\mathrm{N}$ acyltransferase. Appl. Envitron. Microbiol. 78: 7107-7113. 